\title{
Low-Complexity Adaptive Saturated Control of a Class of Nonlinear Systems with Its Application
}

\author{
Gang Zhang, ${ }^{1,2,3}$ Deqiang Cheng $\mathbb{D}^{1}{ }^{1}$ and Qiqi Kou ${ }^{1}$ \\ ${ }^{1}$ School of Information and Control Engineering, China University of Mining and Technology, Xuzhou, Jiangsu, China \\ ${ }^{2}$ Architectural Intelligence College, Jiangsu Vocational Institute of Architectural Technology, Xuzhou, Jiangsu, China \\ ${ }^{3}$ Jiangsu Collaborative Innovation Center for Building Energy Saving and Construction Technology, Xuzhou, Jiangsu, China
}

Correspondence should be addressed to Deqiang Cheng; deqcheng@163.com

Received 25 December 2018; Accepted 27 May 2019; Published 16 June 2019

Academic Editor: Sabri Arik

Copyright (C) 2019 Gang Zhang et al. This is an open access article distributed under the Creative Commons Attribution License, which permits unrestricted use, distribution, and reproduction in any medium, provided the original work is properly cited.

\begin{abstract}
This paper investigates a low-complexity saturated control law for a class of nonlinear systems with consideration of the timevarying output constraint, control constraint, and external disturbance. First, a dead-zone model is employed to transform the control saturation nonlinearity into a linear one with respect to the real input signal. Then, the original system with time-varying output constraint is transformed into a constraint-free one, based on which a novel adaptive saturated control law is devised along the filtered error manifold. By employing minimum learning parameter technique and virtual error concept, only two adaptive parameters are needed to update online, which reduces the computational burdens dramatically. Finally, the applications to DuffingHolmes chaotic system are organized to validate the effectiveness of the proposed control law.
\end{abstract}

\section{Introduction}

In recent years, considerable attention has been paid to developing systematic methods of adaptive control of nonlinear systems including Duffing-Holmes chaotic system and mechatronic system (e.g., see [1-3] and references therein). As a result, extensive interesting works have been carried out in the existing literature. For example, an adaptive neural network (NN)-based control scheme was proposed for perturbed strict-feedback nonlinear systems in [4]. In [5], barrier Lyapunov function (BLF) technique was applied to solve the output control problem for strict feedback system subject to unknown dynamics.

Based on the adaptive control methods in the existing works, one can find that backstepping technique has been widely adopted to develop the relevant control scheme for cascaded nonlinear systems including strict and pure feedback systems. In [6], an adaptive neural control protocol was investigated for a class of unknown pure feedback systems, wherein the unknown nonlinear function is approximated by a radial basis function NN (RBFNN). In terms of nonlinear approximation, fuzzy logic system is also an efficient tool, which is similar with NN. By combing backstepping technique and fuzzy logic system, an effective adaptive fuzzy control scheme was devised for strict feedback system in [7]. However, an inherent drawback, i.e., "explosion of complexity," is often encountered in the backstepping technique owing to the complex computations for the virtual controller and its derivatives $[8,9]$. To conquer this drawback, via introducing a first-order low-pass filter in each step of backstepping controller design, dynamic surface control (DSC) method is proposed to avoid the tedious computations for the derivatives of virtual controller. In this sense, the inherent drawback "explosion of complexity" is conquered to some extent. Thus, DSC method was furthered explored in the nonlinear controller design for strict-feedback systems in [10] and the interconnected nonlinear pure-feedback systems in [11]. Though the computational complexity can be decreased greatly, the computational load in updating the elements of NN or fuzzy logic systems is still very large, especially for high-order nonlinear systems. To conquer this problem, minimal learning parameter (MLP) technique involving less adaptive parameters for strict-feedback systems in [12] provides an alternative way to lower the complexity of adaptive scheme. Thus, how to lower the computational complexity of adaptive control laws deserves further investigations. 
Apart from the aforementioned discussions, in practical engineering systems such as air conditioner, flexible crane, robot, and hypersonic vehicle $[13,14]$, state constraints are also often encountered, which makes it pretty difficult to facilitate the corresponding controller. Among the state constraints, output limitation is one typical constraint which potentially degrades the system performance and easily causes serious hazards. To handle this problem, adaptive BLF-based backstepping control schemes attracted extensive attention in the existing works like in $[5,13]$. As an alternative, considering the prescribed performance of the output originated from [15], [16] investigated a distinctive handling method to transform the time-varying constrained nonaffine system into a constraint-free strict-feedback one. Then, an efficient adaptive control law was developed by employing the NN-based nonlinear approximation. Compared with BLFbased backstepping control laws, the approach proposed by [16] is more advantageous and easily achievable owing to the fact that the recursive design procedure of backstepping technique is avoided. However, the control input under the aforementioned control laws is always very large to guarantee no violation of the output constraints, which is hard to execute in practical systems. Thus, it deserves further investigations on the constrained control for the unknown nonlinear systems.

Motivated by the aforementioned discussions, in this paper, a novel low-complexity adaptive control law is investigated for a class of uncertain nonlinear systems by employing the MLP and RBFNN techniques with consideration of timevarying output constraint, control saturation, and external disturbance. To the authors' best knowledge, there exist very few works reported on the adaptive saturated control for nonlinear systems with time-varying output constraint using MLP technique and virtual error concept. Compared with the existing works, our main contributions are twofold: (1) a novel low-complexity adaptive neural saturated control law is first proposed for a class of nonlinear systems in the presence of time-varying output constraint, control saturation, and external disturbance, wherein the control saturation nonlinearity is approximated by a dead-zone model. (2) The number of adaptive parameters is reduced to only two, which is not dependent on the system order, hidden nodes of $\mathrm{NN}$, and the rules number of fuzzy logic systems. In this case, the computational load is decreased dramatically and the corresponding control law is more easily achievable in practice.

The rest of this paper is organized as follows. Section 2 gives the basic description of the controlled system along with some brief introduction for the RBFNN technique. In Section 3, the proposed low-complexity adaptive saturated law is shown. A group of numerical simulations is organized in Section 4. Some conclusions are given in Section 5.

1.1. Notations. $\mathbb{N}, \mathbb{N}^{+}$represent the set of nonnegative integers and positive integers, respectively. $\mathbb{R}^{n}, \mathbb{R}^{n+}$ are the set of $n$ dimensional real numbers, and $n$-dimensional positive real numbers, respectively. $T$ represents the vector transpose. The Euclidean norm and the absolute value operator are denoted by $\|\cdot\|$ and $|\cdot|$, respectively.

\section{Problem Statement and Preliminaries of RBFNN}

2.1. Model Description. The following system is considered in this paper:

$$
\begin{aligned}
\dot{x}_{i} & =f\left(\bar{x}_{i}\right)+x_{i+1}, \quad i=1,2, \ldots, n-1 \\
\dot{x}_{n} & =f\left(\bar{x}_{n}\right)+b u+d \\
y & =x_{1}
\end{aligned}
$$

where $\bar{x}_{n}=\left[x_{1}, x_{2}, \ldots, x_{n}\right]^{T} \in \mathbb{R}^{n}, y \in \mathbb{R}$ are the state vector and system output, respectively. $\bar{x}_{i}=\left[x_{1}, x_{2}, \ldots, x_{i}\right]^{T} \in \mathbb{R}^{i}$, $f\left(\bar{x}_{i}\right)$ is a continuous nonlinear function with at least $(n-i)$ order derivatives, which is unknown. $b \in \mathbb{R}$ is the known control gain, which is not equal to zero with consideration of the system controllability. $u, d \in \mathbb{R}$ denote the control input and bounded external disturbance, respectively.

In practical engineering applications including robot system and electrical power system, actuator saturation is often encountered. In this work, symmetrical control constraints are considered, i.e., $|u| \leq u_{0}$; consequently, the output of the actuator is given by

$$
u(t)=\operatorname{sat}(v(t))=\operatorname{sign}(v(t)) \min \left\{u_{0},|v(t)|\right\}
$$

where $u_{0}$ is the upper bound of control input. $v(t)$ is the real input of actuator to be determined in the following sections. For brevity, hereafter, $(t)$ is omitted without inducing any ambiguities. To facilitate the controller design, based on [17], saturation nonlinearity in (2) can be transformed into a linear form with respect to $v$, which is

$$
u=\operatorname{sat}(v)=\rho_{0} v-\int_{0}^{\ell} \phi(\tau) d z_{\tau}(v) \mathrm{d} \tau
$$

where $\rho_{0}=\int_{0}^{\ell} \phi(\tau) \mathrm{d} \tau$ is a positive constant, $\phi(\tau)$, a density function, satisfies that $\phi(\tau) \geq 0, \forall \tau>0, \tau>\ell$, and $\phi(\tau)=$ $0\left(\ell \in \mathbb{R}^{+}\right) d z_{\tau}(v)=\max \{v-\tau, \min \{0, v+\tau\}\}$ is the deadzone operator.

Assumption 1. For system (1) subject to input constraint, there exists feasible actual control input $v$; i.e., $v$ is bounded, such that the expected control objective can be achieved.

Remark 2. According to [17], the actual control input $v$ is bounded due to controllability of a practical system. Moreover, many functions can be utilized as the density function including the Gaussian function. Thus, under Assumption 1, the approximation error $-\int_{0}^{\ell} \phi(\tau) d z_{\tau}(v) \mathrm{d} \tau$ is bounded.

Accordingly, substituting (3) into (1) yields

$$
\begin{aligned}
\dot{x}_{i} & =f\left(\bar{x}_{i}\right)+x_{i+1}, \quad i=1,2, \ldots, n-1 \\
\dot{x}_{n} & =f\left(\bar{x}_{n}\right)+\rho_{0} b u+d^{*} \\
y & =x_{1}
\end{aligned}
$$

where $d^{*}:=d-\int_{0}^{\ell} \phi(\tau) d z_{\tau}(v) \mathrm{d} \tau$ is the compound disturbance. Accordingly, the control objective of this work is that 
an expected output reference trajectory $y_{r}$ can be tracked stably with the devised controller. In this case, the output $y$ is evolved within the envelope preplanned by the time-varying asymmetric constraints involving the reference $y_{r}$, i.e.,

$$
\underline{y}(t)<y(t)<\bar{y}(t)
$$

where $y(t), \bar{y}(t)$ denote the lower and upper bounds and satisfy $\bar{y}(t)<y_{r}<\bar{y}(t)$. It is assumed that $y, \bar{y}, y_{r}$ are continuous functions with at least $(n-1)$-order derivatives, which are known and bounded. Note that it is also assumed that the initial output $y(0)$ or $x_{1}(0)$ lies in the envelope described by (5) above.

2.2. Radial Basis Function NN Approximation. In the existing works, a continuous function is often approximated with an arbitrary accuracy over a compact set by a radial basis function NN (RBFNN). For an unknown function $\mathscr{G}(X)$, the output of RBFNN with approximation errors is

$$
\mathscr{G}(X)=W^{* T} \varphi(X)+O(X)
$$

where $X=\left[X_{1}, X_{2}, \ldots, X_{N}\right]^{T} \in \mathbb{R}^{N}$ is the input vector with $N \in \mathbb{N}^{+} . W^{*}=\left[W_{1}^{*}, W_{2}^{*}, \ldots, W_{m}^{*}\right]^{T} \in \mathbb{R}^{m}$ is the optimal weight vector ( $m$ is the node number of hidden layer. $O(X)$ is the approximation error. $\varphi(X)=\left[\varphi_{1}(X)\right.$, $\left.\varphi_{2}(X), \ldots, \varphi_{m}(X)\right]^{T} \in \mathbb{R}^{m}$ with $\varphi_{i}(X)(i=1,2, \ldots, m)$ being a Gaussian kernel function:

$$
\varphi_{i}(X)=\exp \left(-\frac{\left\|X-\gamma_{i}\right\|^{2}}{2 \varepsilon_{i}^{2}}\right)
$$

where $\gamma_{i}=\left[\gamma_{i 1}, \gamma_{i 2}, \ldots, \gamma_{i N}\right]^{T} \in \mathbb{R}^{N}$ and $\varepsilon_{i}$ are, respectively, the relevant center and width of Gaussian functions.

In (6), it is assumed that there is an ideal weight vector $W^{*}$ which makes the approximation error $O(X)$ sufficiently small. Moreover, in order to develop the following adaptive saturated controller, some assumptions are imposed.

Assumption 3. There exists an unknown positive constant $\theta$ which makes condition $\left\|W^{*}\right\| \leq \theta$ hold.

Assumption 4. The unknown external disturbance $d$ is bounded, i.e., $|d| \leq d_{0}$ with $d_{0}$ an unknown positive constant.

Remark 5. Based on Assumption 3, the adaptive scheme to be determined can be developed to estimate the unknown constant $\theta$ rather than the elements of vector $W^{*}$, which decreases the computational load dramatically. This handling way is also referred to as the minimal learning parameter (MLP) scheme [12].

Remark 6. Assumption 4 is reasonable owing to the fact that if the external disturbance is unbounded, system (1) will be out of control under bounded control input.

According to the foregoing discussion, the following work is about the design of the relevant adaptive saturated controller in the presence of unknown dynamics and output constraints.

\section{Main Results}

3.1. Output Transformation. As (5) shows, the output is constrained, which will increase the difficulty and complexity of designing an efficient controller directly. In order to surmount this problem, the following output transformation is employed, which is

$$
y=\mathscr{P}\left(z_{1}, \underline{y}, \bar{y}\right)
$$

or

$$
z_{1}(t)=\mathscr{P}^{-1}(y, \underline{y}, \bar{y})
$$

where $z_{1}$ is the transformed output variable. Function $\mathscr{P}$ should satisfy the following conditions:

$$
\begin{gathered}
\frac{\partial \mathscr{P}(\cdot)}{\partial z_{1}}>0, \\
\lim _{z_{1} \longrightarrow-\infty} \mathscr{P}\left(z_{1}, \underline{y}, \bar{y}\right)=\underline{y}(t), \\
\lim _{z_{1} \longrightarrow+\infty} \mathscr{P}\left(z_{1}, \underline{y}, \bar{y}\right)=\bar{y}(t)
\end{gathered}
$$

Inspired by [16], function $\mathscr{P}$ is selected as

$$
\mathscr{P}\left(z_{1}, \underline{y}, \bar{y}\right)=\frac{\bar{y}-\underline{y}}{2} \tanh \left(z_{1}\right)+\frac{\bar{y}+\underline{y}}{2}
$$

Then, based on (11), the transformed output $z_{1}$ equals

$$
z_{1}=\operatorname{atanh}\left(\frac{2 y-\bar{y}-\underline{y}}{\bar{y}-\underline{y}}\right)
$$

Accordingly, let $z_{2}=\dot{z}_{1}, z_{3}=\dot{z}_{2}=\ddot{z}_{1}, \ldots, z_{n}=\dot{z}_{n-1}=z_{1}^{(n-1)}$; then one can obtain that

$$
\begin{aligned}
& \dot{z}_{i}=z_{i+1}, \quad i=1,2, \ldots, n-1 \\
& \dot{z}_{n}=\beta\left(f(\bar{x})+\rho_{0} b v+d^{*}\right)+g(Y)
\end{aligned}
$$

with

$$
\begin{aligned}
g(Y):= & \sum_{k=1}^{n-1}\left(\begin{array}{c}
n-1 \\
k
\end{array}\right)\left[\frac{\partial \mathscr{P}^{-1}(\cdot)}{\partial y}\right]^{(k)} \cdot y^{(n-k)} \\
& +\sum_{k=0}^{n-1}\left(\begin{array}{c}
n-1 \\
k
\end{array}\right)\left[\frac{\partial \mathscr{P}^{-1}(\cdot)}{\partial \underline{y}}\right]^{(k)} \cdot \underline{y}^{(n-k)} \\
& +\sum_{k=0}^{n-1}\left(\begin{array}{c}
n-1 \\
k
\end{array}\right)\left[\frac{\partial \mathscr{P}^{-1}(\cdot)}{\partial \bar{y}}\right]^{(k)} \cdot \bar{y}^{(n-k)}
\end{aligned}
$$

where $Y:=[y, \underline{y}, \bar{y}]^{T} \cdot \beta:=\partial \mathscr{P}^{-1}(\cdot) / \partial y=2 /\left(1-\alpha^{2}\right)>0$ with $\alpha:=(2 y-\bar{y}-\underline{y}) /(\bar{y}-\underline{y}) .\left(\begin{array}{c}n-1 \\ k\end{array}\right)$ is the binomial coefficient.

3.2. Adaptive Saturated Controller Design. To proceed, based on transformed system (13), the following filtered state variable is defined as

$$
s=c_{1} z_{1}+c_{2} z_{2}+\cdots+c_{n-1} z_{n-1}+z_{n}
$$


where there exists a positive constant vector $c:=\left[c_{1}, c_{2}\right.$, $\ldots, 1]^{T} \in \mathbb{R}^{n}$ such that multinomial $\lambda^{n}+c_{n-1} \lambda^{n-1}+\cdots+c_{2} \lambda^{2}+$ $c_{1}=0$ is Hurwitz with $\lambda$ being the Laplace operator [18].

Lemma 7 (see [19]). For the filtered state variable in (15), if $s$ converges to zero as $t \longrightarrow \infty$, then the newly defined tracking error $z_{1}$ and its derivatives $z_{k}(k=2, \ldots, n)$ converge to zero with the same convergence rate as $t \longrightarrow \infty$.

Proof. Inspired by [19], an equivalent transformation of (15) is expressed by

$$
\begin{gathered}
s(t)=\dot{\chi}_{n-1}(t)+\omega_{n-1} \chi_{n-1}(t) \\
\chi_{n-1}(t)=\dot{\chi}_{n-2}(t)+\omega_{n-2} \chi_{n-2}(t) \\
\vdots \\
\chi_{2}(t)=\dot{\chi}_{1}(t)+\omega_{1} \chi_{1}(t)=\dot{z}_{1}(t)+c_{1} z_{1}(t) \\
\chi_{1}(t)=z_{1}(t)
\end{gathered}
$$

where parameter $\omega_{i}$ is associated with $c_{1}, c_{2}, \ldots, c_{n}$ in (15). Then solving the first equation in (16) yields

$$
\begin{aligned}
\chi_{n-1}(t)= & \chi_{n-1}(0) \exp \left(-\omega_{n-1} t\right) \\
& +\exp \left(-\omega_{n-1} t\right) \int_{0}^{t} s(\tau) \exp \left(\omega_{n-1} \tau\right) \mathrm{d} \tau
\end{aligned}
$$

Equation (17) implies that if term $\int_{0}^{t} s(\tau) \exp \left(\omega_{n-1} \tau\right) \mathrm{d} \tau$ is bounded, then $\chi_{n-1}(t) \longrightarrow 0$ as $t \longrightarrow \infty$. If term $\int_{0}^{t} s(\tau) \exp \left(\omega_{n-1} \tau\right) \mathrm{d} \tau$ is unbounded, for (17), employing L'Hopital's rule can yield

$$
\begin{aligned}
\lim _{t \rightarrow \infty} \chi_{n-1}(t) & =0+\lim _{t \rightarrow \infty} \frac{s(t) \exp \left(\omega_{n-1} t\right)}{\omega_{n-1} \exp \left(\omega_{n-1} t\right)} \\
& =\lim _{t \rightarrow \infty} \frac{s(t)}{\omega_{n-1}}
\end{aligned}
$$

Accordingly, when $s(t) \longrightarrow 0$ as $t \longrightarrow \infty, \chi_{n-1}(t) \longrightarrow 0$ as $t \longrightarrow \infty$ with the same convergence rate as $s(t)$. Likewise, for the second equation in (16), based on (18), employing L'Hopital's rule can yield

$$
\begin{aligned}
\lim _{t \rightarrow \infty} \chi_{n-2}(t) & =0+\lim _{t \rightarrow \infty} \frac{\chi_{n-1}(t) \exp \left(\omega_{n-2} t\right)}{\omega_{n-2} \exp \left(\omega_{n-2} t\right)} \\
& =\lim _{t \rightarrow \infty} \frac{\chi_{n-1}(t)}{\omega_{n-2}}=\lim _{t \rightarrow \infty} \frac{s(t)}{\omega_{n-1} \omega_{n-2}}
\end{aligned}
$$

As shown in (19), if $s(t) \longrightarrow 0$ as $t \longrightarrow \infty, \chi_{n-2}(t) \longrightarrow$ 0 as $t \longrightarrow \infty$ with the same convergence rate as $s$. For the remaining equations in (16), by employing the same procedure like (17)-(19), one can obtain the same conclusion. Thereby, the proof of Lemma 7 is completed.

Taking the time derivative of $s$ yields

$$
\begin{aligned}
\dot{s} & =\dot{z}_{n}+\sum_{i=1}^{n-1} c_{i} z_{i+1} \\
& =f^{*}\left(\bar{x}_{n}, \underline{y}, \bar{y}\right)+\beta \rho_{0} b v+\beta d^{*}+\sum_{i=1}^{n-1} c_{i} z_{i+1}
\end{aligned}
$$

where $f^{*}\left(\bar{x}_{n}, y, \bar{y}\right):=\beta f\left(\bar{x}_{n}\right)+g(Y)+\sum_{i=1}^{n-1} c_{i} z_{i+1}$. Owing to unknown nonlinearity, $f\left(\bar{x}_{n}\right)$ is unknown as well. Thus, according to the preliminaries in Section 2.2, RBFNN is utilized to approximate the lumped unknown function $f^{*}$, which is given by

$$
f^{*}\left(Y^{*}\right)=W^{T} \varphi\left(Y^{*}\right)
$$

where $Y^{*}:=\left[\bar{x}_{n}^{T}, y, \bar{y}\right]^{T} \in \mathbb{R}^{n+2}$. According to Assumption 3 above, one can obtain that

$$
\begin{aligned}
\left|f^{*}\right| & =\left\|f^{*}\right\|=\left\|W^{T} \varphi\left(Y^{*}\right)\right\| \leq\left\|W^{T}\right\|^{2}+\frac{\left\|\varphi\left(Y^{*}\right)\right\|^{2}}{4} \\
& \leq \theta^{2}+\frac{\left\|\varphi\left(Y^{*}\right)\right\|^{2}}{4} \leq \vartheta_{1} \psi_{1}
\end{aligned}
$$

where $\vartheta_{1}:=\max \left\{\theta^{2}, 1 / 4\right\}, \psi_{1}:=1+\left\|\varphi\left(Y^{*}\right)\right\|^{2} / 4$. For the compound disturbance $d^{*}$, under Remark 2 and Assumption 4, one can obtain

$$
\begin{aligned}
\left|\beta d^{*}\right| & =\beta\left|d^{*}\right| \leq \beta\left(|d|+\left|\int_{0}^{\ell} \phi(\tau) d z_{\tau}(v) \mathrm{d} \tau\right|\right) \\
& \leq \vartheta_{2} \psi_{2}
\end{aligned}
$$

where $\psi_{2}:=\beta$ with $\beta>0$ a time-varying positive parameter given in (14). $\vartheta_{2}$ is an unknown positive constant. Based on the foregoing analysis, the adaptive saturated controller is devised as

$$
\begin{aligned}
v & =-\frac{1}{\beta \rho_{0} b}(\eta s \\
& +\left(\widehat{\vartheta}_{1}+\frac{\mu_{1}}{1+\exp \left(-\int_{0}^{t} \widehat{\vartheta}_{1}(\tau) \mathrm{d} \tau\right)}\right) \psi_{1} \operatorname{sign}(s) \\
& \left.+\left(\widehat{\vartheta}_{2}+\frac{\mu_{2}}{1+\exp \left(-\int_{0}^{t} \widehat{\vartheta}_{2}(\tau) \mathrm{d} \tau\right)}\right) \psi_{2} \operatorname{sign}(s)\right)
\end{aligned}
$$


where $\eta, \mu_{1}, \mu_{2}$ are positive constants. $\widehat{\vartheta}_{1}, \widehat{\vartheta}_{2}$ are, respectively, the estimated values of unknown constants $\vartheta_{1}, \vartheta_{2}$. The corresponding adaptive scheme is

$$
\begin{aligned}
& \dot{\widehat{\vartheta}}_{1}=-\frac{\mu_{1} \exp \left(-\int_{0}^{t} \widehat{\vartheta}_{1}(\tau) \mathrm{d} \tau\right)}{\left(1+\exp \left(-\int_{0}^{t} \widehat{\vartheta}_{1}(\tau) \mathrm{d} \tau\right)\right)^{2}} \widehat{\vartheta}_{1}+\psi_{1}|s| \\
& \dot{\bar{\vartheta}}_{2}=-\frac{\mu_{2} \exp \left(-\int_{0}^{t} \widehat{\vartheta}_{2}(\tau) \mathrm{d} \tau\right)}{\left(1+\exp \left(-\int_{0}^{t} \widehat{\vartheta}_{2}(\tau) \mathrm{d} \tau\right)\right)^{2}} \widehat{\vartheta}_{2}+\psi_{2}|s|
\end{aligned}
$$

Remark 8. The controller (24) has two parts. One is the nominal part: $-\left(1 / \beta \rho_{0} b\right) \eta s$ which is used to stabilize the tracking error system. The other one is the robust part, i.e., $-\left(1 / \beta \rho_{0} b\right)\left(\widehat{\vartheta}_{1}+\mu_{1} /\left(1+\exp \left(-\int_{0}^{t} \widehat{\vartheta}_{1}(\tau) \mathrm{d} \tau\right)\right)\right) \psi_{1} \operatorname{sign}(s)$ and $-\left(1 / \beta \rho_{0} b\right)\left(\widehat{\vartheta}_{2}+\mu_{2} /\left(1+\exp \left(-\int_{0}^{t} \widehat{\vartheta}_{2}(\tau) \mathrm{d} \tau\right)\right)\right) \psi_{2} \operatorname{sign}(s)$ which is utilized to further compensate the effects brought by the unknown nonlinearity, external disturbance, and control saturation. Compared with [16], only two parameters are needed to estimate rather than $2 m$ parameters involved in the weight vector of RBFNN approximation. In this sense, the computational load is decreased dramatically.

3.3. Stability Analysis. Under the devised controller (24), an important result is given as follows.

Theorem 9. Under the designed controller (24) and adaptive scheme (25), the desired output reference command $y_{r}$ evolved within the envelope preplanned by the time-varying constraints can be tracked with asymptotic stability.

Proof. First, construct the following Lyapunov function as

$$
V=V_{1}+V_{2}
$$

with

$$
\begin{aligned}
& V_{1}=\frac{1}{2} s^{2}, \\
& V_{2}=\frac{1}{2}\left(\widetilde{\vartheta}_{1}^{2}+\widetilde{\vartheta}_{2}^{2}\right)
\end{aligned}
$$

where the virtual error $\widetilde{\vartheta}_{i}$ is defined as $\widetilde{\vartheta}_{i}:=\mathcal{\vartheta}_{i}-\left(\widehat{\vartheta}_{i}+\mu_{i} /(1+\right.$ $\left.\left.\exp \left(-\int_{0}^{t} \widehat{\vartheta}_{i}(\tau) \mathrm{d} \tau\right)\right)\right)(i=1,2)$ which is inspired by [19]. Taking the time derivative of $V_{1}$ in (27) yields

$$
\dot{V}_{1}=s \dot{s}
$$

Substituting (20) and (24) into (28) yields

$$
\begin{aligned}
\dot{V}_{1} & =s\left(f^{*}\left(\overline{\mathbf{x}}_{n}, \underline{y}, \bar{y}\right)+\beta \rho_{0} b v+\beta d^{*}\right) \\
& =-\eta s^{2}-\left(\widehat{\vartheta}_{1}-\frac{\mu_{1}}{1+\exp \left(-\int_{0}^{t} \widehat{\vartheta}_{1}(\tau) \mathrm{d} \tau\right)}\right) \psi_{1}|s|
\end{aligned}
$$

$$
\begin{aligned}
& -\left(\widehat{\vartheta}_{2}+\frac{\mu_{2}}{1+\exp \left(-\int_{0}^{t} \widehat{\vartheta}_{2}(\tau) \mathrm{d} \tau\right)}\right) \psi_{2}|s| \\
+ & s\left(f^{*}\left(\overline{\mathbf{x}}_{n}, \underline{y}, \bar{y}\right)+\beta d^{*}\right)-\eta s^{2} \\
& -\left(\widehat{\vartheta}_{1}-\frac{\mu_{1}}{1+\exp \left(-\int_{0}^{t} \widehat{\vartheta}_{1}(\tau) \mathrm{d} \tau\right)}\right) \psi_{1}|s| \\
& -\left(\widehat{\vartheta}_{2}+\frac{\mu_{2}}{1+\exp \left(-\int_{0}^{t} \widehat{\vartheta}_{2}(\tau) \mathrm{d} \tau\right)}\right) \psi_{2}|s|+|s| \\
& \cdot\left|f^{*}\left(\overline{\mathbf{x}}_{n}, \underline{y}, \bar{y}\right)+\beta d^{*}\right| \\
\leq & -\eta s^{2}-\left(\widehat{\hat{\vartheta}_{1}}-\frac{\mu_{1}}{1+\exp \left(-\int_{0}^{t} \widehat{\vartheta}_{1}(\tau) \mathrm{d} \tau\right)}\right) \psi_{1}|s| \\
& -\left|f^{*}\left(\bar{x}_{n}, \underline{y}, \bar{y}\right)\right|+|s| \cdot\left|\beta d^{*}\right| \\
& \left(\hat{\vartheta}_{2}+\frac{\mu_{2}}{1+\exp \left(-\int_{0}^{t} \widehat{\vartheta}_{2}(\tau) \mathrm{d} \tau\right)}\right) \psi_{2}|s|+|s|
\end{aligned}
$$

In view of (22) and (23), (29) equals

$$
\begin{aligned}
\dot{V}_{1} \leq & -\eta s^{2}-\left(\widehat{\vartheta}_{1}-\frac{\mu_{1}}{1+\exp \left(-\int_{0}^{t} \widehat{\vartheta}_{1}(\tau) \mathrm{d} \tau\right)}\right) \psi_{1}|s| \\
& -\left(\widehat{\vartheta}_{2}+\frac{\mu_{2}}{1+\exp \left(-\int_{0}^{t} \widehat{\vartheta}_{2}(\tau) \mathrm{d} \tau\right)}\right) \psi_{2}|s| \\
& +\vartheta_{1} \psi_{1}|s|+\vartheta_{2} \psi_{2}|s|
\end{aligned}
$$

Taking the time derivative of $V_{2}$ in (27) yields

$$
\begin{aligned}
\dot{V}_{2}= & -\widetilde{\vartheta}_{1}\left(\dot{\hat{\vartheta}}_{1}+\frac{\mu_{1} \exp \left(-\int_{0}^{t} \widehat{\vartheta}_{1}(\tau) \mathrm{d} \tau\right)}{\left(1+\exp \left(-\int_{0}^{t} \widehat{\vartheta}_{1}(\tau) \mathrm{d} \tau\right)\right)^{2}} \widehat{\vartheta}_{1}\right) \\
& -\widetilde{\vartheta}_{2}\left(\dot{\hat{\vartheta}}_{2}+\frac{\mu_{2} \exp \left(-\int_{0}^{t} \widehat{\vartheta}_{2}(\tau) \mathrm{d} \tau\right)}{\left(1+\exp \left(-\int_{0}^{t} \widehat{\vartheta}_{2}(\tau) \mathrm{d} \tau\right)\right)^{2}} \widehat{\vartheta}_{2}\right)
\end{aligned}
$$

Substituting (30) and (31) into the time derivative of $V$ in (26) yields

$$
\begin{aligned}
\dot{V} \leq & -\eta s^{2}-\left(\widehat{\vartheta}_{1}-\frac{\mu_{1}}{1+\exp \left(-\int_{0}^{t} \widehat{\vartheta}_{1}(\tau) \mathrm{d} \tau\right)}\right) \psi_{1}|s| \\
& -\left(\widehat{\vartheta}_{2}+\frac{\mu_{2}}{1+\exp \left(-\int_{0}^{t} \widehat{\vartheta}_{2}(\tau) \mathrm{d} \tau\right)}\right) \psi_{2}|s|
\end{aligned}
$$




$$
\begin{aligned}
& -\widetilde{\vartheta}_{1}\left(\dot{\hat{\vartheta}}_{1}+\frac{\mu_{1} \exp \left(-\int_{0}^{t} \widehat{\vartheta}_{1}(\tau) \mathrm{d} \tau\right)}{\left(1+\exp \left(-\int_{0}^{t} \widehat{\vartheta}_{1}(\tau) \mathrm{d} \tau\right)\right)^{2}} \widehat{\vartheta}_{1}\right) \\
& -\widetilde{\vartheta}_{2}\left(\dot{\hat{\vartheta}}_{2}+\frac{\mu_{2} \exp \left(-\int_{0}^{t} \widehat{\vartheta}_{2}(\tau) \mathrm{d} \tau\right)}{\left(1+\exp \left(-\int_{0}^{t} \widehat{\vartheta}_{2}(\tau) \mathrm{d} \tau\right)\right)^{2}} \widehat{\vartheta}_{2}\right) \\
& +\vartheta_{1} \psi_{1}|s|+\vartheta_{2} \psi_{2}|s|
\end{aligned}
$$

Based on the definition of virtual error $\widetilde{\vartheta}_{i}(i=1,2)$ in (27), then (32) becomes

$$
\begin{aligned}
\dot{V} & \leq-\eta s^{2}+\left(\vartheta_{1}\right. \\
& \left.-\left(\widehat{\vartheta}_{1}-\frac{\mu_{1}}{1+\exp \left(-\int_{0}^{t} \widehat{\vartheta}_{1}(\tau) \mathrm{d} \tau\right)}\right)\right) \psi_{1}|s|+\left(\vartheta_{2}\right. \\
& \left.-\left(\widehat{\vartheta}_{2}+\frac{\mu_{2}}{1+\exp \left(-\int_{0}^{t} \widehat{\vartheta}_{2}(\tau) \mathrm{d} \tau\right)}\right)\right) \psi_{2}|s| \\
& -\widetilde{\vartheta}_{1}\left(\dot{\hat{\vartheta}}_{1}+\frac{\mu_{1} \exp \left(-\int_{0}^{t} \widehat{\vartheta}_{1}(\tau) \mathrm{d} \tau\right)}{\left(1+\exp \left(-\int_{0}^{t} \widehat{\vartheta}_{1}(\tau) \mathrm{d} \tau\right)\right)^{2}} \widehat{\vartheta}_{1}\right) \\
& -\widetilde{\vartheta}_{2}\left(\dot{\hat{\vartheta}}_{2}+\frac{\mu_{2} \exp \left(-\int_{0}^{t} \widehat{\vartheta}_{2}(\tau) \mathrm{d} \tau\right)}{\left(1+\exp \left(-\int_{0}^{t} \widehat{\vartheta}_{2}(\tau) \mathrm{d} \tau\right)\right)^{2}} \widehat{\vartheta}_{2}\right) \\
& \leq-\eta s^{2}+\widetilde{\vartheta}_{1}\left(\psi_{1}|s|\right. \\
& \left.-\left(\tilde{\hat{\vartheta}}_{2}+\frac{\mu_{2} \exp \left(-\int_{0}^{t} \widehat{\vartheta}_{2}(\tau) \mathrm{d} \tau\right)}{\left(1+\exp \left(-\int_{0}^{t} \widehat{\vartheta}_{2}(\tau) \mathrm{d} \tau\right)\right)^{2}} \widehat{\vartheta}_{2}\right)\right) \\
& \left.-\left(\dot{\hat{\vartheta}}_{1}+\frac{\mu_{1} \exp \left(-\int_{0}^{t} \widehat{\vartheta}_{1}(\tau) \mathrm{d} \tau\right)}{\left(1+\exp \left(-\int_{0}^{t} \widehat{\vartheta}_{1}(\tau) \mathrm{d} \tau\right)\right)^{2}} \widehat{\vartheta}_{1}\right)\right) \\
& (
\end{aligned}
$$

Substituting (25) into (33) yields

$$
\dot{V} \leq-\eta s^{2} \leq 0
$$

Only when $s=0$ can $\dot{V}=0$. Thus, the tracking error system is asymptotically stable, i.e., $s \longrightarrow 0$ as $t \longrightarrow \infty$. Accordingly,

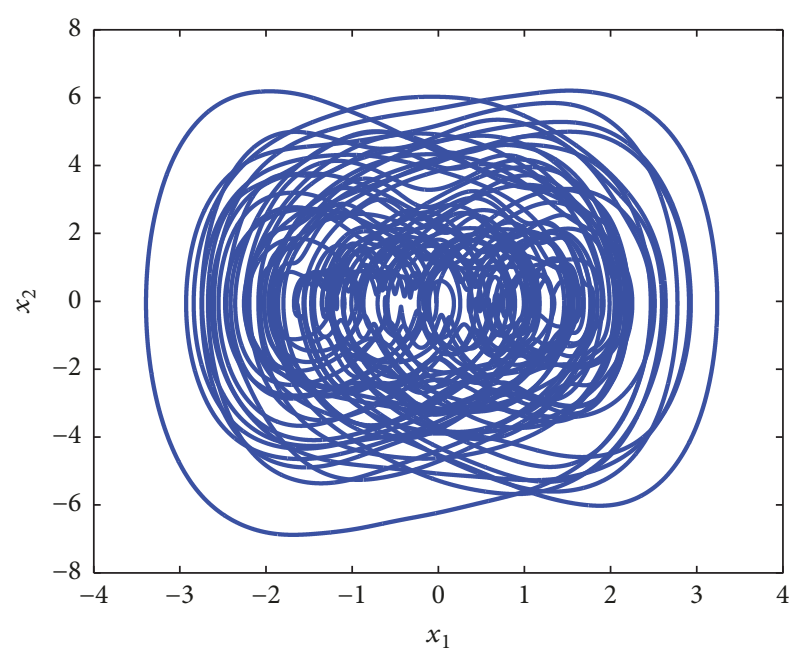

FIGURE 1: Phase image of system (35) without control input.

based on (15), by employing Lemma 7 , one can obtain that all the newly defined tracking errors $z_{1}, z_{2}, \ldots, z_{n}$ converge to zero as $t \longrightarrow \infty$. This completes the proof of Theorem 9 .

\section{Illustrative Example}

To validate the effectiveness of the proposed control law, simulations of a second-order Duffing Holmes chaotic system are employed. In this application example, the chaotic system is expressed by [20]

$$
\begin{aligned}
\dot{x}_{1} & =x_{2} \\
\dot{x}_{2} & =-\omega_{1} x_{1}-\omega_{2} x_{2}-\omega_{3} x_{1}^{3}+q \cos (\xi t)+\operatorname{sat}(u)+d \\
y & =x_{1}
\end{aligned}
$$

where $\omega_{1}=0.3+0.2 \sin (10 t), \omega_{2}=0.2+0.2 \cos (5 t)$, $\omega_{3}=1, q=5+0.1 \cos (t), \xi=0.5+0.1 \sin (t)$, $|u| \leq 10, d=0.4 \sin (0.2 \pi t)+0.3 \sin \left(x_{1} x_{2}\right)$. The phase image of Duffing Holmes chaotic system is demonstrated in Figure 1. According to Remark 2, Gaussian kernel function with detailed form given in (7) can be utilized as one part of the density function. When the density function is selected as $\phi=10 \exp \left(\tau^{2} / 2\right)$, one can obtain $\rho_{0}=12.5$. The simulation parameters are set: $y_{r}=\sin (0.5 t)+\cos (0.25 t), \bar{y}=y_{r}+$ $\exp (-0.3 t)+0.45, y=y_{r}-\exp (-0.3 t)-0.45$ in (5); $m=$ $50, \varepsilon_{i}=0.5+2 \operatorname{rand}(\cdot), \gamma_{i}=[2,-8,6,2]^{T}(i=1,2, \ldots, m)$ in (6) and (7); $c_{1}=2, \eta=100, \mu_{1}=\mu_{2}=3, \widehat{\vartheta}_{1}(0)=$ $5+\operatorname{rand}(\cdot), \widehat{\vartheta}_{2}(0)=3+\operatorname{rand}(\cdot)$ in (15) and (24). In order to show the effectiveness of the proposed control law, the traditional backstepping control method is employed as the comparative test (the detail introduction can be found in [6], which is omitted for brevity). The number of nodes of RBFNN is 50 with the center evenly spaced on $[-1,3] \times[-17,1] \times$ $[-5,17] \times[-2,6] \times[-5,7] \times[-4,10]$. The initial weights $W_{b}=$ $-3+6$ rand (.) of the RBFNN in the traditional backstepping 


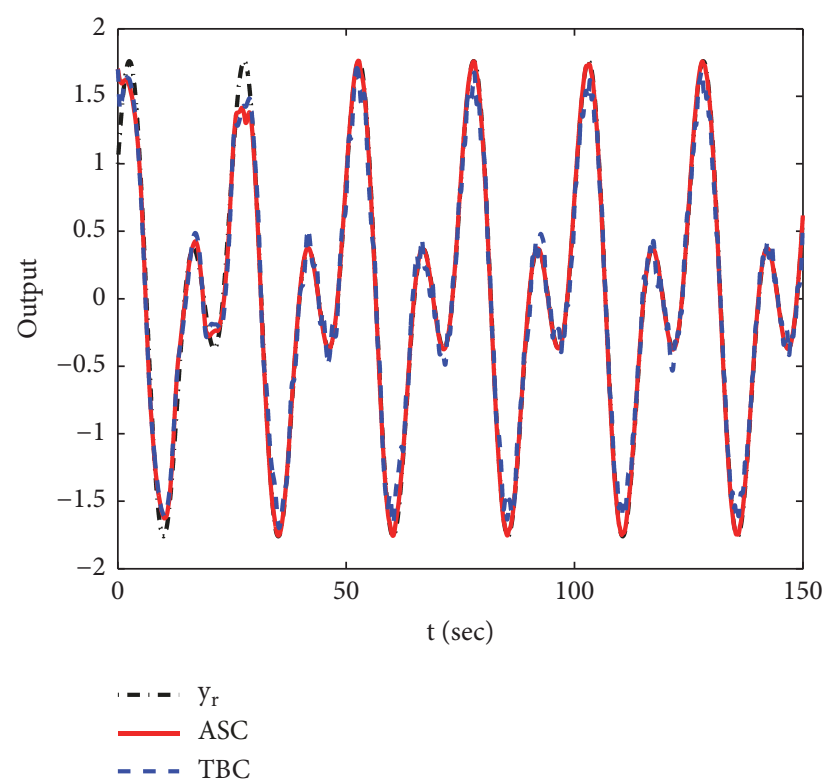

FIGURE 2: Output tracking trajectories under two control laws.
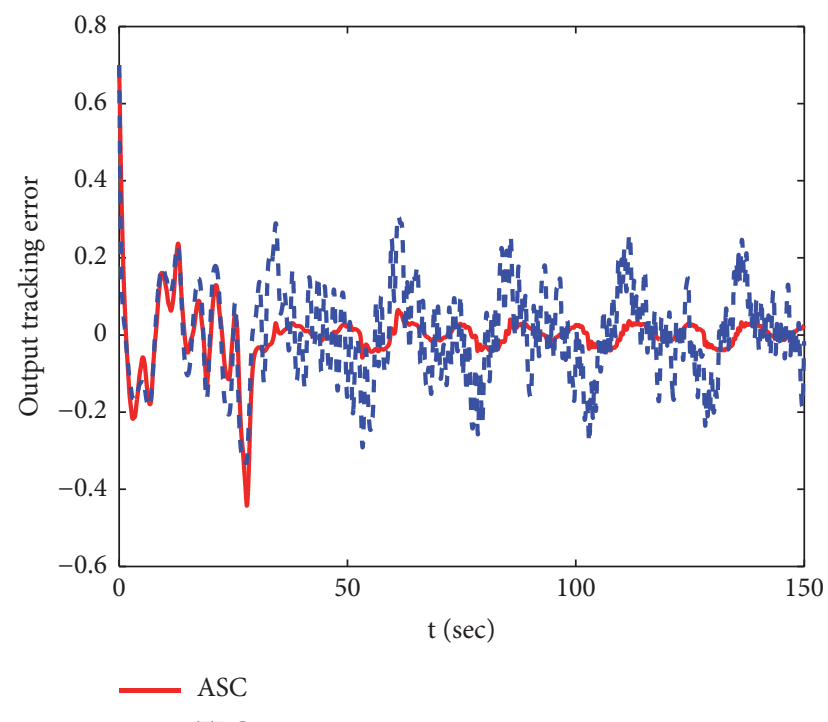

FIGURE 3: Output tracking errors under two control laws.
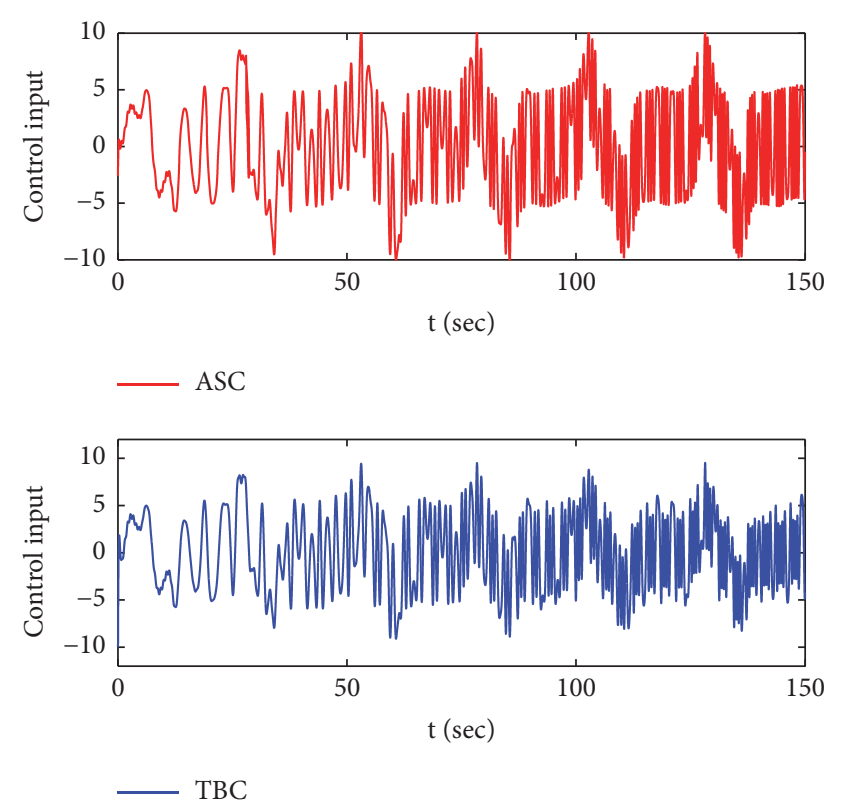

FIGURE 4: Control input $u=\operatorname{sat}(v)$ under two control laws.

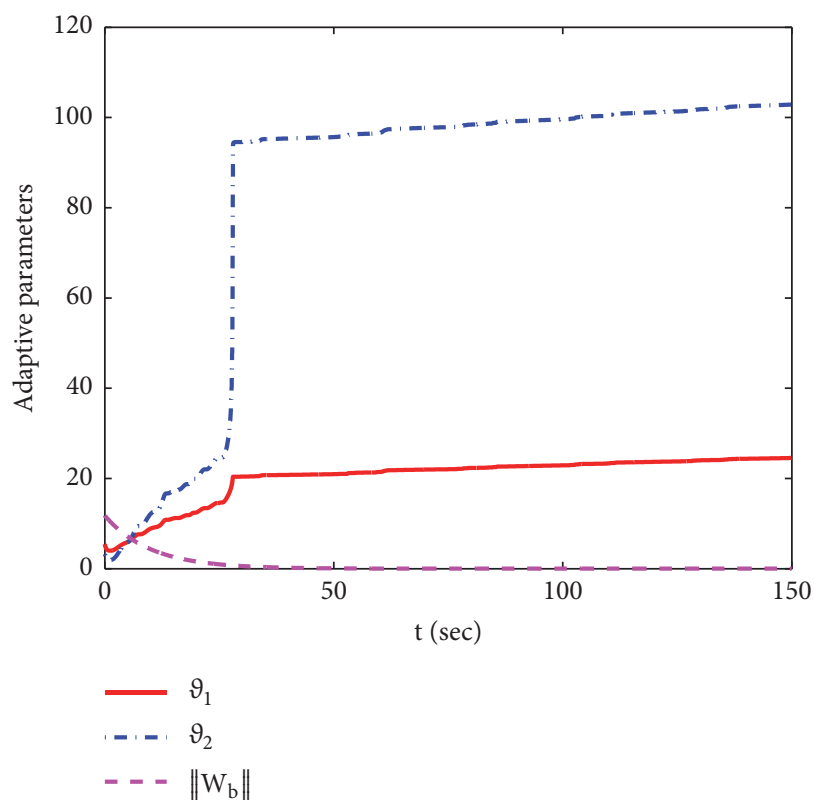

Figure 5: Adaptive parameters under two control laws.

backstepping control law. In this sense, the computational load is decreased dramatically.

\section{Conclusion}

In this paper, a novel low-complexity adaptive saturated control law has been investigated for a class of nonlinear systems with consideration of time-varying output constraint, control saturation, and external disturbance. Compared with the existing works, the computational load of adaptive schemes is very small and the asymptotic stability of tracking error 


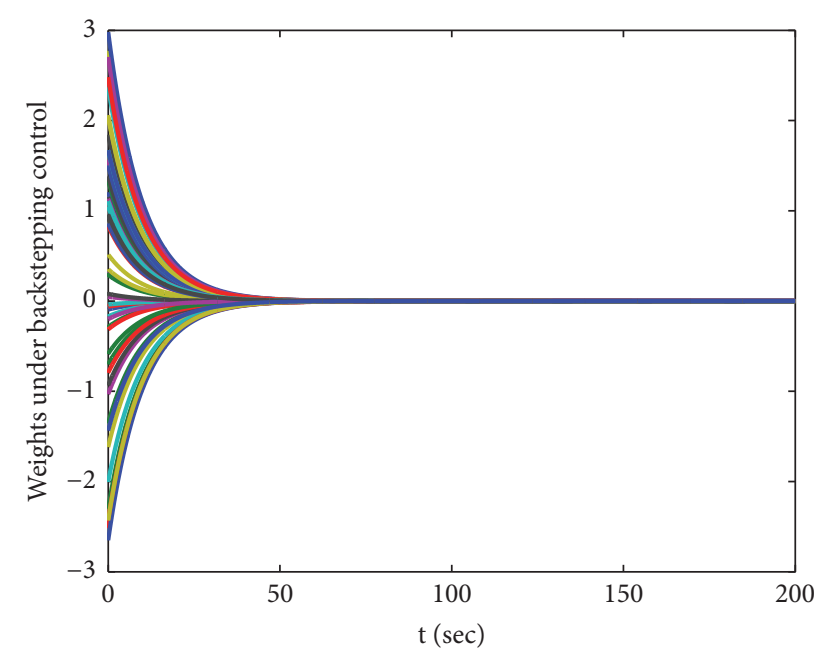

Figure 6: Weights $W_{b}$ of RBFNN under traditional backstepping control law.

system can be guaranteed rather than the uniformly ultimately bounded stability. The employed simulations show that about 4 times improvement can be obtained in the tracking accuracy, which demonstrates the effectiveness of the proposed control law.

\section{Data Availability}

The data used to support the findings of this study are included within the article.

\section{Conflicts of Interest}

The authors declare that they have no conflicts of interest.

\section{Acknowledgments}

This work was supported by the National Natural Science Foundation of China (Grant no. 51774281), Doctor Fund of Jiangsu Collaborative Innovation Center for Building Energy Saving and Construction Technology (Grant SJXTBS1702), Technical Projects of Jiangsu Construction System (Grants 2016ZD58 and 2017ZD006), and Scientific Project of Xuzhou (Grant KH17001).

\section{References}

[1] S. Garcia, "Hopf bifurcations drops in the lid-driven square cavity flow," Advances in Applied Mathematics and Mechanics, vol. 1, no. 4, pp. 546-572, 2009.

[2] K. Dehmani, F. Fourati, K. Elleuch, and A. Toumi, "Multiple neural control strategies using a neuro-fuzzy classifier," Automatic Control and Computer Sciences, vol. 52, no. 3, pp. 155-165, 2018.

[3] B. Zhang, J. Yuan, L. Qiu, N. Cheung, and J. F. Pan, "Distributed coordinated motion tracking of the linear switched reluctance machine-based group control system," IEEE Transactions on Industrial Electronics, vol. 63, no. 3, pp. 1480-1489, 2016.
[4] S. S. Ge and J. Wang, "Adaptive NN tracking control of uncertain nonlinear discrete-time systems with nonaffine deadzone input," IEEE Transactions on Neural Networks, vol. 45, no. 3, pp. 497-505, 2017.

[5] B. Ren, S. S. Ge, K. P. Tee, and T. H. Lee, "Adaptive neural control for output feedback nonlinear systems using a barrier lyapunov function," IEEE Transactions on Neural Networks and Learning Systems, vol. 21, no. 8, pp. 1339-1345, 2010.

[6] C. Wang, D. J. Hill, S. S. Ge, and G. Chen, "An ISS-modular approach for adaptive neural control of pure-feedback systems," Automatica, vol. 42, no. 5, pp. 723-731, 2006.

[7] A.-M. Zou, Z.-G. Hou, and M. Tan, "Adaptive control of a class of nonlinear pure-feedback systems using fuzzy backstepping approach," IEEE Transactions on Fuzzy Systems, vol. 16, no. 4, pp. 886-897, 2008.

[8] T. S. Li, D. Wang, G. Feng, and S. C. Tong, "A DSC approach to robust adaptive $\mathrm{NN}$ tracking control for strict-feedback nonlinear systems," IEEE Transactions on Systems, Man, and Cybernetics, Part B: Cybernetics, vol. 40, no. 3, pp. 915-927, 2010.

[9] C. Wei, J. Luo, H. Dai, Z. Yin, and J. Yuan, "Low-complexity differentiator-based decentralized fault-tolerant control of uncertain large-scale nonlinear systems with unknown dead zone," Nonlinear Dynamics, vol. 89, no. 4, pp. 2573-2592, 2017.

[10] D. Wang and J. Huang, "Neural network-based adaptive dynamic surface control for a class of uncertain nonlinear systems in strict-feedback form," IEEE Transactions on Neural Networks and Learning Systems, vol. 16, no. 1, pp. 195-202, 2005.

[11] Y. M. Li, S. C. Tong, and T. S. Li, "Adaptive fuzzy output feedback dynamic surface control of interconnected nonlinear pure-feedback systems," IEEE Transactions on Cybernetics, vol. 45, no. 1, pp. 138-149, 2015.

[12] Y. Yang and J. Ren, "Adaptive fuzzy robust tracking controller design via small gain approach and its application," IEEE Transactions on Fuzzy Systems, vol. 11, no. 6, pp. 783-795, 2003.

[13] W. He, S. Zhang, and S. S. Ge, "Adaptive control of a flexible crane system with the boundary output constraint," IEEE Transactions on Industrial Electronics, vol. 61, no. 8, pp. 41264133, 2014.

[14] C. Wei, J. Luo, H. Dai, and J. Yuan, "Efficient adaptive constrained control with time-varying predefined performance for a hypersonic flight vehicle," International Journal of Advanced Robotic Systems, vol. 14, no. 2, p. 1729881416687504, 2017.

[15] C. P. Bechlioulis and G. A. Rovithakis, "Adaptive control with guaranteed transient and steady state tracking error bounds for strict feedback systems," Automatica, vol. 45, no. 2, pp. 532-538, 2009.

[16] W. Meng, Q. Yang, and Y. Sun, "Adaptive neural control of nonlinear MIMO systems with time-varying output constraints," IEEE Transactions on Neural Networks and Learning Systems, vol. 26, no. 5, pp. 1074-1085, 2015.

[17] M. Chen, P. Shi, and C.-C. Lim, "Robust constrained control for MIMO nonlinear systems based on disturbance observer," Institute of Electrical and Electronics Engineers Transactions on Automatic Control, vol. 60, no. 12, pp. 3281-3286, 2015.

[18] A. Bartoszewicz, "A new reaching law for sliding mode control of continuous time systems with constraints," Transactions of the Institute of Measurement and Control, vol. 37, no. 4, pp. 515-521, 2015.

[19] Y. Song, Y. Wang, and C. Wen, "Adaptive fault-tolerant pi tracking control with guaranteed transient and steady-state performance," Institute of Electrical and Electronics Engineers 
Transactions on Automatic Control, vol. 62, no. 1, pp. 481-487, 2017.

[20] M. Chen and S. S. Ge, "Direct adaptive neural control for a class of uncertain nonaffine nonlinear systems based on disturbance observer," IEEE Transactions on Cybernetics, vol. 43, no. 4, pp. 1213-1225, 2013. 


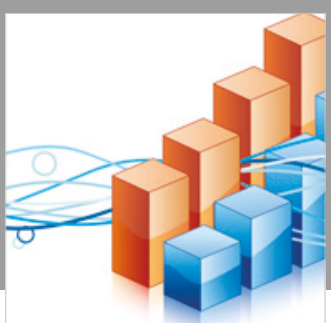

Advances in

Operations Research

\section{-n-m}
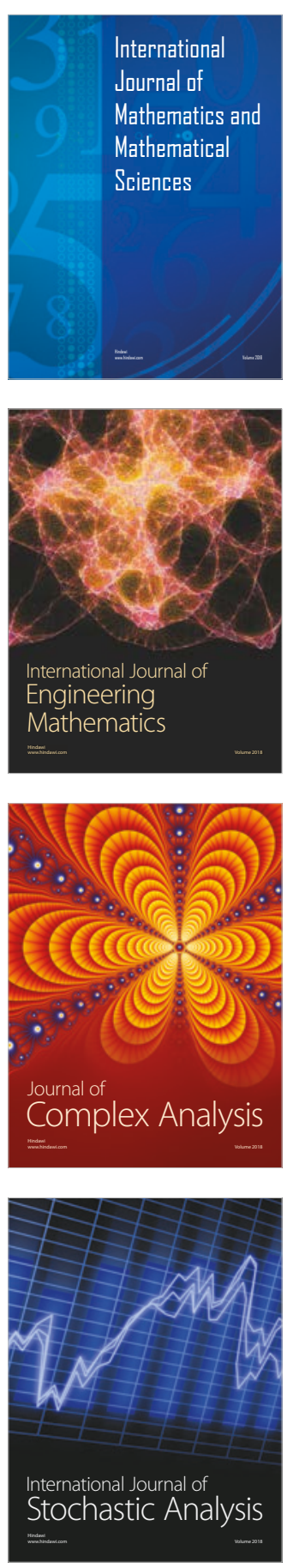
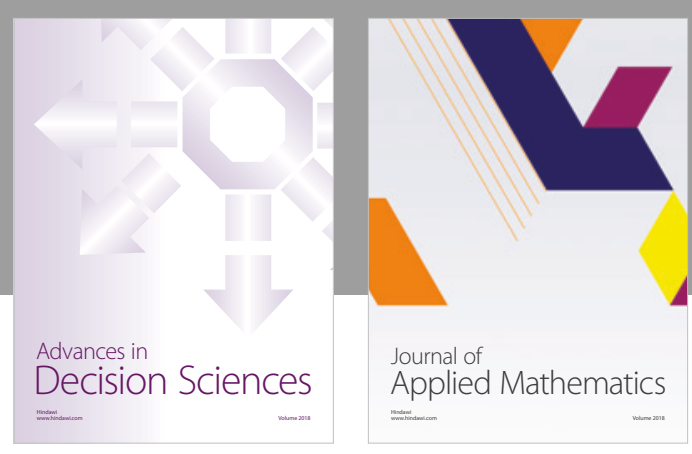

Journal of

Applied Mathematics
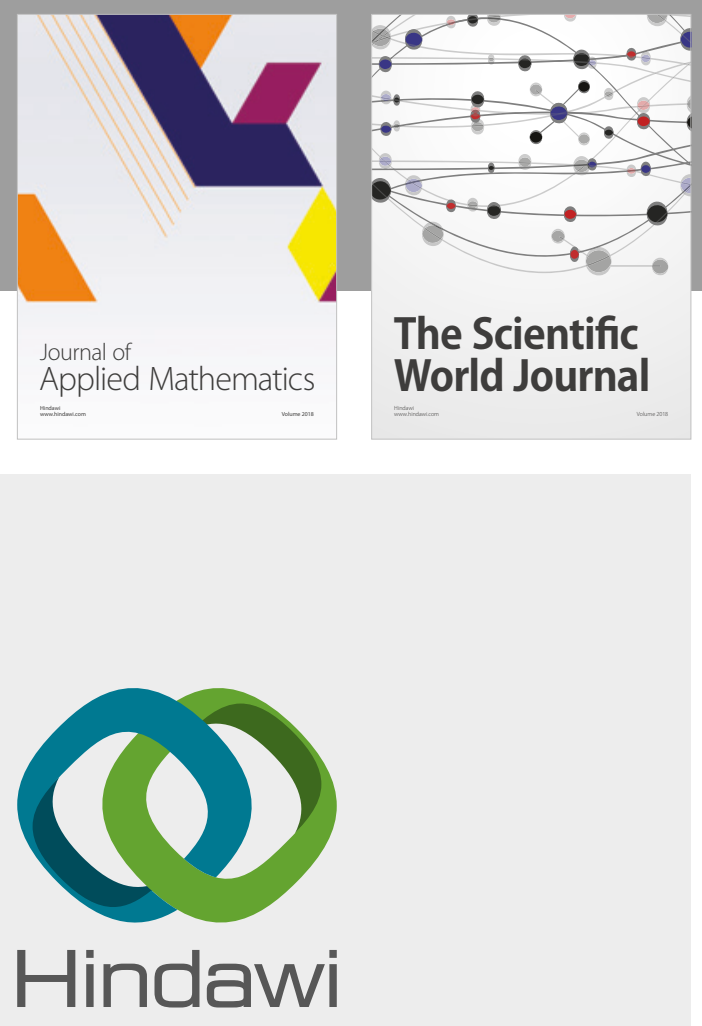

Submit your manuscripts at

www.hindawi.com

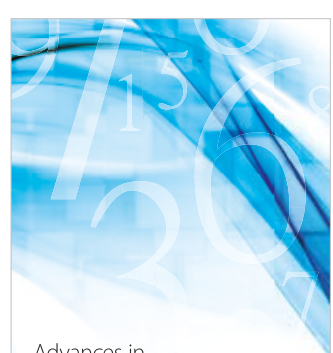

Advances in
Numerical Analysis
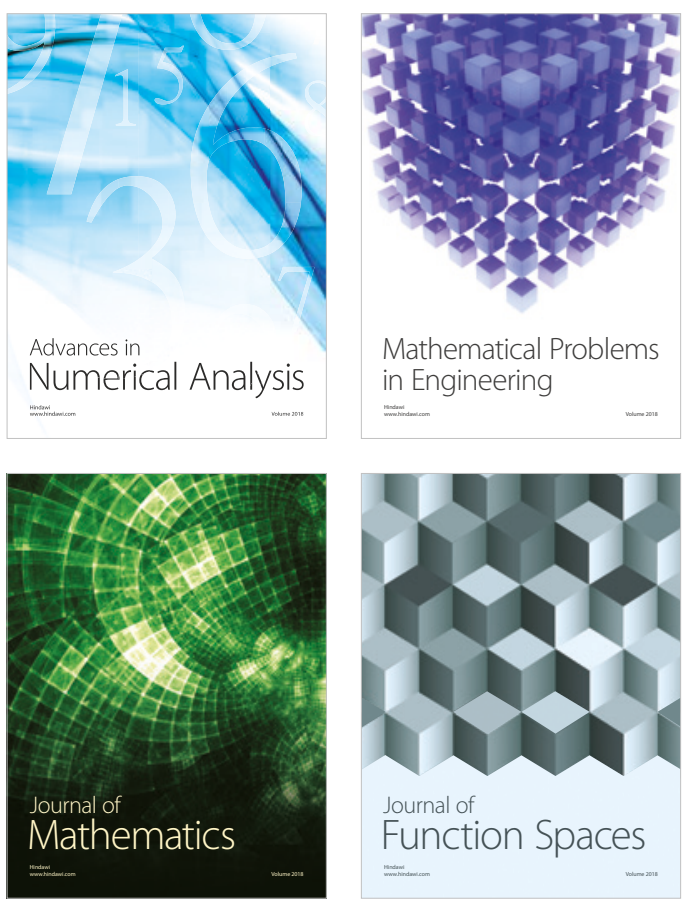

Mathematical Problems in Engineering

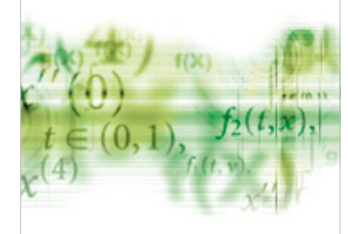

International Journal of

Differential Equations

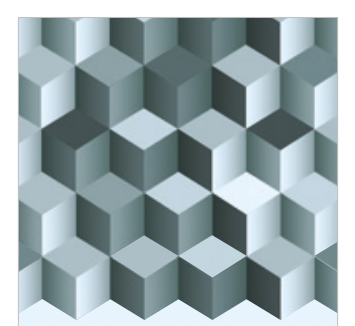

Journal of

Function Spaces

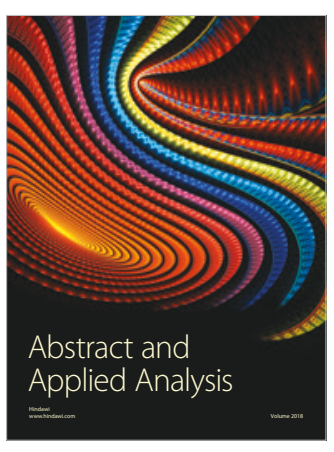

The Scientific

World Journal

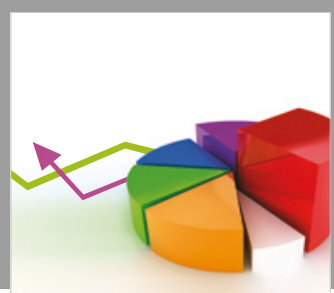

Journal of

Probability and Statistics
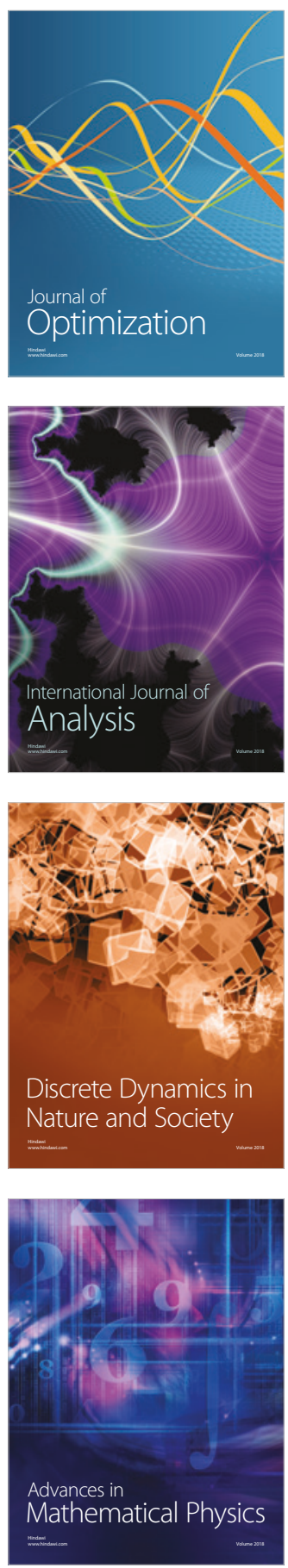